\title{
Cognitive Implications of Musical Perception
}

\author{
Adam Lockhart \\ Center for Experimental Music and Intermedia, Division of Composition Studies, College of \\ Music, University of North Texas, email: adam12@unt.edu
}

\begin{abstract}
Any musical experience is innately pertinent to cognition and psychology. This paper discusses various aspects of musical cognition, including notions of timbre in relation to spectromorphology, a term coined by Denis Smalley, a notable writer on aesthetics of electroacoustic music. Furthermore, a cognitive model will serve to explain inherent interrelationships involving multi-modal perception.
\end{abstract}

\section{PERCEPTION OF MUSICAL MOTION}

Motion in music is realized through perception. It is perceived as a temporally progressive event that involves three elements: 1) a continuous perceptual history of a piece of music, 2) the now constantly being organized by both the conscious and the subconscious mind, and 3) projections into the future drawn from connections made by the conscious and subconscious mind. Occurrence of perception in a musical context engages everything in our musical perceptual history, or personal environment while simultaneously redefining it (Lockhart, Keller 2006). This is a process through which we may render expectations (while also redefining the basis for which we may render expectations in the future). Perception of motion in music is an abstraction of physical motion in a musical context.

One type of musical motion in electroacoustic music, texture motion, involves tendencies that render a sense of direction as an abstraction of change in spectral mass -Agglomeration (an accumulation of spectral mass), and Dissipation (a dispersing or disintegrating mass) (Smalley 1997). This vocabulary provides for ideas of motion that are slightly more complex than ascending or descending patterns of frequency, which are more immediately directional.

\section{COGNITION IN THE IMMEDIATE}

The now is simply a notion of the present, and for the purposes of this paper it involves cognitive activities unfolding within some imperceptible temporal proximity, a transitory cache of the fleeting recent, the temporally immediate, and some alignment with the future. The subconscious mind is always organizing sensory information. Once something is generalized further abstractions are organized by making associations. While in a broad sense they represent an expansive network of meaning, associations that come from these cognitive activities subsequently may be regarded individually as thought entities: bundled generalizations.

\subsection{Source Bonding as a Perceptual Cue}

Perceptual cues are something like flags that are raised when associative connections are made subconsciously. Source bonding represents one type of perceptual cue signifying a cognitive connection made associating sound and sound source. When referring to a note or several different notes played on an instrument the idea is very clear-cut. Smalley's definition of source bonding could easily be the foundation for defining timbre in terms of "the timbre of an instrument":

(Source bonding is) the natural tendency to relate sounds to supposed sources and causes, and to relate sounds to each other because they appear to have shared or associated origins. (Smalley 1994)

However, in electronic music timbre is much more complex. There are even problems with this case of an instrument having timbre. If the instrument has a timbre, then why is it that the instrumentalist can get different timbres out of the instrument? Why does it have a different timbre in a different room? Why does the recording of that instrument in a room have timbral variation when you play it on different stereo systems? The idea of source bonding coming from a perceptual cue is a better foundation for a discussion of timbre that takes a cognitive approach.

\section{TIMBRE}

A thorough definition of timbre should involve dispelling inadequate notions of timbre. I propose that timbre is not some notion of characteristic spectral qualities, but that the term more significantly defines some cognitive process. Before discussing Smalley's definition of timbre we must adopt the term spectromorphology as a description of any sound in terms of the vertical and horizontal aspects of music:

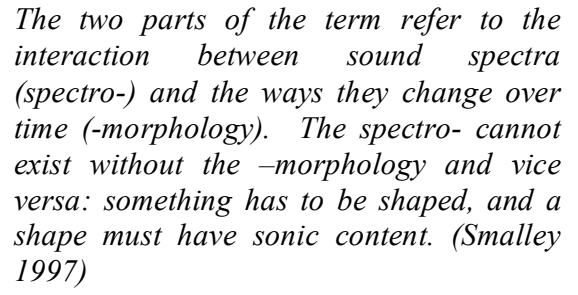

The term spectromorphology is well adapted for the purposes of discussing any music or sound. At the same time, the term itself describes the very nature of sound and the fundamental elements therein. Spectral profile of sound over time becomes important in the following discussion of timbre.

\subsection{Definition of Timbre}

Discussion of timbre involves tiptoeing through a discursive minefield. Definition of timbre (along with music itself and music technology) has evolved over centuries. The ASA (American Standards Association) defined timbre as the quality that makes sounds of equal pitch and loudness different (ASA 1960). This does not satisfy in discussion of spectrum over time. Smalley has adapted a definition for timbre in terms of spectromorphology, "a general, sonic physiognomy 
whose spectromorphological ensemble permits the attribution of identity." (Smalley 1994). Identity is not always some result of source bonding. Any given sound object's identity comes from its spectromorphological profile -- perceived attributes that can be recognized. Also a sound object could have source bonding with some imaginary source. Therefore timbre must be a flexible notion involving cognition and recognition.

It is inadequate to think of timbre as some spectral signature, but it is identity, a perceptual cue denoting a neatly packaged bundle of abstractions that defines a sound object by its spectromorphological boundaries, that truly defines timbre. Consider now the spectromorphology of a tree falling in a forest... Does it have timbral identity? Perhaps if you imagine such a spectromorphology, in your mind it does have timbral identity -- even if it never actually happened.

\subsection{Layered Identities}

Any audiophile will tell you that every single component of an audio reproduction system has its own unique timbre, and any violin player will tell you that every violin has its own unique timbre. Likewise, an audio engineer will say the same of every concert hall. This is to say that one sound may result in a multitude of timbral identities.

To explain in detail, on one level, a violin is a violin is a violin. On another level, this is this violin and that is that violin. Everything is dependent on the identities of which one makes oneself aware. One may be aware of this violin in this hall on these microphones ... (and so on) ... this Compact Disc transport through this digital cable to this DAC interconnects - preamp - more interconnects - amp - speaker cables - speakers and finally the room, room treatments and positioning of the speakers in the room all have their own unique timbre. This is, of course, only if one is aware; otherwise, the components do not have a multitude of layered identities. Another possible layer of timbre in electroacoustic music could be the timbre of certain processes. Comb filters, time expansions, pitch shifts, filters and granulations are just a few examples of processes that may have timbral identities to the careful ear. This explains further that timbre is a function of perception.

Identity, for the listener, is the result of consciously engaging perceptual cues: connections made unconsciously. It should be noted that in order to listen for any of these timbres one must "train the ear". This really means that one's mind must build a meaningful network of associations relative to the particular listening experience.

\section{GESTURE}

The fundamentals of a sounding gesture are rooted in a sourcecause bond whereby a human energy motion trajectory excites a sounding body and creates spectromorphological consequences (Smalley 1997). Source-cause bonding allows for an inferred notion of physical gestural activity. The subconscious mind contains a wealth of psychophysical information. This information is some subsection of one's network of meaning and useful knowledge that is engaged during the musical experience. This is a tactile sense that one need not act on, but people often breathe along with swells of musical energy without even realizing that they are physically aligning themselves with the music.

\begin{abstract}
From the viewpoint of both (sounding) agent and watching listener, the musical gesture-process is tactile and visual, as well as aural. Moreover, it is proprioceptive: that is, it is concerned with the tension and relaxation of muscles, with effort and resistance. In this way, soundmaking is linked to more comprehensive sensorimotor and psychological experience. (Smalley 1997)
\end{abstract}

The experience of the watching listener is therefore multimodal including visual and aural sensory modes as well as some tactile interpretation. Likewise, even if a listener is not watching but perhaps listening to a recording, the experience is still multi-modal. Gesture can be inferred or felt from almost any music and realized in an abstract tactile sense.

\subsection{Psychophysical Re-enactment}

Feeling involves a rather unusual subdivision into two distinct species of meaning: physical and emotional. This is an interesting dichotomy considering that both physical and emotional feelings relate to psychophysical information (some mental relationship with the physical qualities of any environment). For example, emotions are generally expressed in a physical manner. Be it crying, yelling, laughing, kissing, or even just a look in someone's eye, emotions engage psychophysical information for means of expression. Conversely, emotion or emotional meaning is perceived, related to psychophysical information and manifested physically or physiologically. Because emotions are communicated through physical expression and music similarly is expressive on some abstract physical level, it is reasonable that music is a medium through which humankind so persistently tries to communicate emotional meaning.

\section{PERCECPTUAL FOCUS}

Perceptual focus can be best described as a function of the conscious mind as it relates to subconscious processes. For example, one might hear music without listening to it. You step into an elevator with music playing. If the conscious effort were made to perceive the content of what was heard, then connections made by the subconscious mind could be mulled over by the conscious mind: "Gosh, that soprano sax really comes through on that 3 " full-range driver." On the other hand you could be distracted and never consciously elaborate on connections being made by the subconscious mind. Later on, you might never even realize how a song you don't like very much got stuck in your head.

\subsection{Reduced Listening}

Reduced listening is a phenomenon may occur upon repetition of a sound event. A mesmerizing example of reduced listening involves the repetition of a verbal utterance. If one were to speak a word (try a funny sounding word) over and over and again, eventually the word would lose its meaning and one would start to hear how funny it sounds. The repetition causes the conscious mind to lose focus of the word's extrinsic connectedness then shift focus more towards the utterance's presence in spectral-space over time. 
Reduced listening comes about through concentrated, repeated listening to a sound event, a common activity in the electroacoustic composing process. It is an investigative process whereby detailed spectro-morphological attributes and relationships are uncovered. (Smalley 1997)

This marks a shift in perceptual focus under which a sound event loses its extrinsic associative qualities and then even expressive qualities of its musical context. I contend that this shift in perceptual focus is exactly the same as the mind shifting towards the first stage of hypnosis.

Because of repetition, few new perceptual cues are made. When the conscious mind exhausts these cues it rests, waiting for more. Then one is able to understand the sound event as a more basic element: spectral motion. In the following examples I intend to show that motion is understood unconsciously. Keep in mind that this example of repetition can be used to understand reduced listening, while it is not essential in order to engage this mode of listening. Once again, as with anything we may listen for, we must train the ear to do so.

Most everyone has experienced this first stage of hypnosis even without being aware. Highway hypnosis exemplifies the first stage of hypnosis, light trance. Anyone who has ever driven down a highway for some period of time is likely to slip into a light trance (and sometimes wonder how they missed their exit).

It is interesting to note that we know how to drive an automobile subconsciously. Another example is someone who is so deep in thought that they wonder how they even stopped properly at a red light. Even more amusing, is the motorist who stops at a green light. The reason that we are able to know how to drive subconsciously is because physical information we collect from our environment (such as motion), the mental representation of which is psychophysical information, is a function of our unconscious minds. This is the reason reduced listening, a shift towards perception of spectral motion, is a shift towards the subconscious. Figure 1. shows a sound event repeating over time, alongside which is a solid line indicating perceptual focus. The line indicates a shift in perceptual focus from a conscious alert state to a more subconscious hypnotic state.

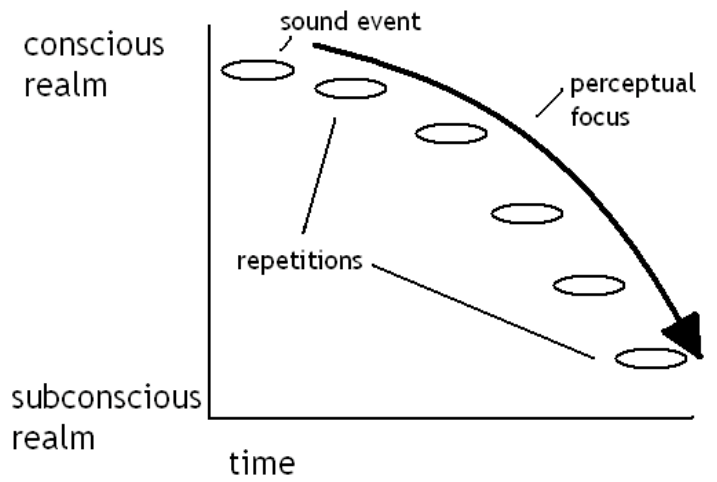

Figure 1. Conscious state over time.

Now consider the Buddhist mantra. Mantra meditation, from Hinduism and Buddhism, is a form of meditation whereby words or phrases are repeatedly chanted internally or out loud as objects of contemplation. Here we find the same idea: repetition of a sound event. This paper assumes that meditation and hypnosis are both psychologically similar forms of trance. One final example of sonic phenomenon inducing trance is from riding a train. The sound a train makes in travel is constant yet it is also repetitious. Listening to the sound of a train can make one drowsy and even help one fall asleep. These examples may aid in understanding the notion of shift in perceptual focus.

\section{A COGNITIVE MODEL}

It is clear that a cognitive model, which allows for all of these inner workings to coexist, is necessary to further this discussion. It is important in the communication of these ideas to have some kind of representation (even if it is some kind of abstract representation that symbolizes something that doesn't physically exist). I propose a cognitive model that offers a way to explain an organized network of knowledge (other than just by those terms), a way to incorporate reduced listening and the idea of a hypnotic state into the model, and provide for the idea of perceptual cues.

\subsection{Generalization Unit Theory}

Every sensory modality is handled in a different part of the brain. For example, visual information is processed by the visual cortex. This example refers to modularity. A module is a set of automatic processes that occurs apart from other cognitive systems (Kellogg, 2003). If all sensory modalities are modulated separately then they must become unified on another level of perceptual processing. Michel Chion applies the term synchresis to describe "the spontaneous and irresistible weld produced between a particular auditory phenomenon and visual phenomenon when they occur at the same time." (Chion 1994) As discussed, such a bond could also include inherent physical information. This paper proposes a generalization unit theory to describe any mental representation of perceived stimuli, wherein all modalities utilize the same trans-modal unit for the purpose of explaining synchresis as well as other strange audio-visual interactions.

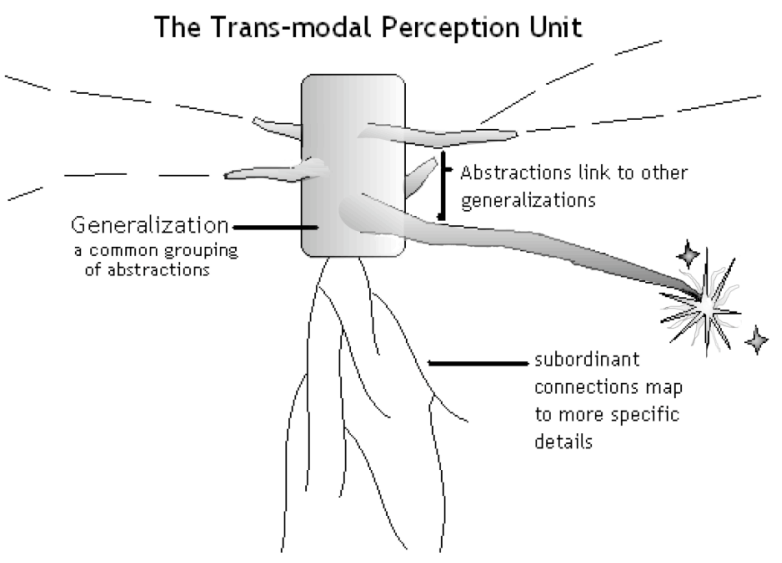

Figure 2. A basic unit of perception.

Figure 2. is a visual representation of a thought entity. It is merely a contrivance to the aid in explaining something that is difficult to imagine having a physical form at all. So it is more realistic to think of this visual representation as expressing some occurrence rather than as an object. The bulk of this unit 
represents a generalization. A generalization is a group of abstractions. Abstractions, similar to generalizations in essence, describe here a single basic component of a generalization that can easily form associations with other abstractions from other generalization units. A generalization here maintains a broader scope, a further level of abstractedness rendering a unified embodiment of that perception. The generalization unit can provide access to pertinent specific details. These details are by nature abstractions as well, but they exclusively belong to a specific generalization. If one were to consciously delve into those specific details and make new connections to other perceptions, they will have effectively turned some detail into a networkable abstraction to make the connection. Generalization units only connect to other generalizations through a network of abstractions. Every thought or perception embodies abstraction.

\subsection{State of Consciousness Cognitive Model}

The State of Consciousness Cognitive Model is contrived in order to explain the interactive process of music cognition. The network of trans-modal perception units at the bottom of Figure 3. represents one's personal environment. This diagram shows only one trans-modal perception unit being introduced into the network. When engaged in a musical experience, however, since each unit represents a generalization made of sensory information it is likely that this model would be at a constant state of accumulation.

First, new information is introduced into the working memory. Working memory attempts to make associations within the network. If an association is made then that connection creates a perceptual cue. Perceptual focus, in Figure 3, is represented as the part that breaks up the straight line from working memory to conscious elaboration. If the perceptual cue does end up in the conscious elaboration stage, then whatever connection was made to create a perceptual cue will be made evident to the conscious mind. This is to suggest that connections are made in your head before they pop up in your conscious mind. Acquirement is learning. It entails a modification of the network of knowledge.

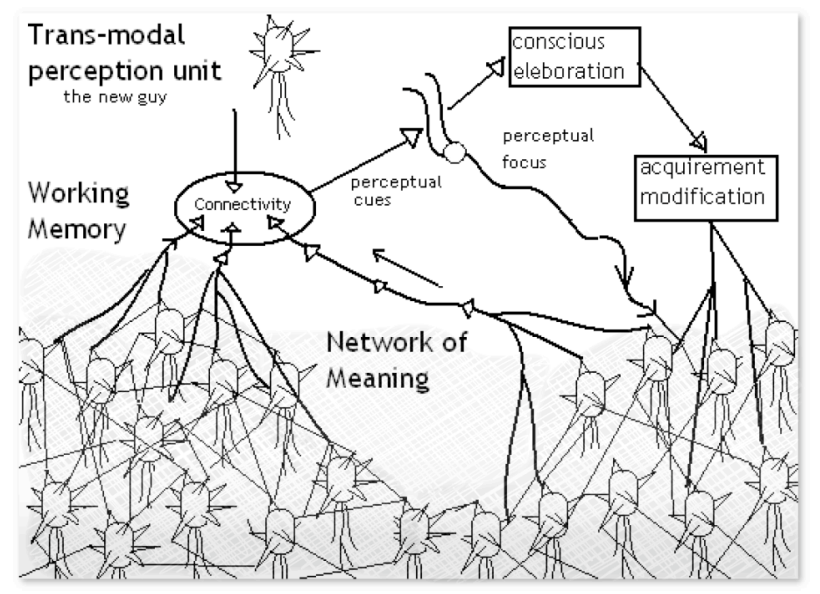

Figure 3. A cognitive model.

Perceptual focus is an aspect of the psyche. Its normal function, a conscious state of awareness, would be to actively seek out perceptual cues as they are created. Recall Figure 1. where perceptual focus is viewed within the context of conscious and subconscious states of awareness. It is important to understand that these are not fixed states, but perceptual focus will generally reside in between the two, to varying degrees. In order to achieve a subconscious state of inner awareness one must quite the mind and be free of perceptual cues. This basically means that perceptual cues are like thoughts that pop up all the time. If one were to relax one's perceptual focus, then instead of sensory generalization units traveling normally to the conscious mind, this model has a pathway (through perceptual focus in its subconscious state) straight to the subconscious mind. In support of this model, consider the suggestibility of the subconscious mind. This open pathway, unfiltered by the conscious mind, could explain such a phenomenon. This model is appropriate for both describing musical experience (including reduced listening) and a model for the cognitive processes involved in hypnotic induction.

\section{CONCLUSION}

Adopting a cognitive model to conceptualize musical perception may be useful for composers who wish to obtain a deeper understanding of their craft. New music such as electroacoustic music, painted on a canvas of spectrum over time, relies on these ideas of cognitive connectivity to evoke substance. When we become immersed in a musical experience, we are actually immersed in our own minds. Music is a profound communication. A composer may hypnotize, cause the heart to pound, amuse or bring one to tears. It is important to be aware of these cognitive aspects in order to communicate most effectively.

\section{REFERENCES}

[1] ASA (American Standards Association) 1960. Acoustical Terminology. American Standards Association, New York.

[2] Chion, M. 1994. Audio-Vision: Sound on Screen. Columbia University Press.

[3] Grow, G. 1996. Serving the Strategic Reader: Cognitive Theory and Its Implications for the Teaching of Writing, Available on-line at: $<\mathrm{http}: / /$ www.longleaf.net/ggrow $>$.

[4] Keller, D., A. Capasso. 2006. New Concepts and Techniques in Eco-Composition. Organized Sound (11)1, 55-62.

[5] Kellogg, R. 2003. Cognitive Psycholgy. Sage Publications.

[6] Lockhart, A., D. Keller. 2006. Exploring Cognitive Process Through Music Composition. Proceedings of the International Computer Music Conference 2006.

[7] Smalley, D. 1994. Defining Timbre - Refining Timbre. Contemporary Music Review (10)2, 35-48.

[8] Smalley, D. 1997. Spectromorphology: Explaining sound-shapes. Organised Sound (2)2, 107-126. 